\title{
Pilot Study: Two-Stage Hybrid Model to Correlate Single Energy CT and PET in Pancreatic Adenocarcinoma
}

\author{
Min Zhang ${ }^{1}$, Jorge Oldan², Miao He ${ }^{1}$, Teresa Wu ${ }^{1,2 *}$, Alvin Silva ${ }^{2}$, Jing Li ${ }^{1}$, J Ross Mitchell ${ }^{1,2}$, William M Pavlicek ${ }^{2}$, Michael C Roarke ${ }^{2}$, and \\ Amy K Hara ${ }^{2}$
}

${ }^{1}$ School of Computing, Informatics, and Decision Systems Engineering, Ira Fulton Schools of Engineering, Arizona State University, Tempe, Arizona, USA ${ }^{2}$ Department of Radiology, Mayo Clinic College of Medicine, Scottsdale, Arizona, USA

\begin{abstract}
Pancreas adenocarcinoma is one of the most common malignant tumors and the fourth leading cause of cancerrelated mortality. While Computed Tomography (CT) has been commonly used clinically for the cancer staging and follow-up, Positron Emission Tomography (PET) is known to be generally more accurate and sensitive for metastases and thus has great prognostic value. However, PET is more expensive and less accessible. This research is to explore the use of multivariate models to extract valuable information from CT to mimic the effects of PET. Based on the original 6 CT measures, 10 CT biomarkers are derived. The strongest correlation with PET SUV in the multivariate regression on the 6 original measures is $r^{2}=0.41(r=0.64)$, on the 10 derived biomarkers is $r^{2}=0.55(r=0.74)$. We developed a twostage hybrid model, where a multivariate classifier was developed to first separate the patients into the group with high SUV values vs. low SUV values, then the regression model was developed for each group respectively. The overall performance of this two-stage model is more promising with an $r^{2}=0.81(r=0.90)$. We conclude advanced CT analytics has the potential to extract valuable information that correlates with PET SUV.
\end{abstract}

Rationale and objectives: Pancreatic adenocarcinoma is commonly studied by CT and PET. We aimed to see if information from CT could be used to simulate the results of PET.

Materials and methods: A retrospective study of 24 patients with pancreatic cancer who had both CT and PET in close temporal proximity was conducted. Measurements of the aorta, normal pancreatic tissue, solid and cystic portions of pancreatic tumors were performed resulting in 6 biomarkers. Ten more biomarkers were derived including the ratios of solid and cystic tumor mean and standard deviation to normal pancreas (and to each other), as well as signal-to-noise ratios of solid and cystic tumors to normal pancreas. Univariate analysis and multivariate regression were conducted on the original measures ( 6 biomarkers) and derived measures (10 biomarkers). A two-stage hybrid model integrating machine learning model with multivariate regression analysis was also studied.

Results: The best results were obtained using the two-stage hybrid model. The regression model for low SUV $(\leq 5)$ used cystic tumor mean $\left(r^{2}=0.68, r=0.83\right)$. The regression model for high SUV $(>5)$ used tumor mean, the ratios of tumor mean to pancreas mean, tumor mean to aorta mean, standard deviation of tumor to aorta mean and signal-to-noise ratio of difference between the normal pancreas mean and solid tumor mean to standard deviation of pancreas $\left(r^{2}=0.86\right.$, $r=0.93)$. The overall performance of the two-stage model is $r^{2}=0.81(r=0.90)$.

Conclusion: Two-stage multivariate analysis of CT parameters can mimic the effects of PET to a reasonable extent, and signal-to-noise and standard deviation ratios may capture the essential nonlinearity of these relationships.

Keywords: Pancreatic adenocarcinoma; Multiple regression; CT; Pancreas; Informatics

\section{Introduction}

Pancreatic adenocarcinoma is the second most common malignant tumor of the gastrointestinal tract and the fourth leading cause of cancer-related mortality accounting for $5 \%$ of cancer-related deaths in the United States [1]. It is estimated that 46,420 new cases of pancreatic adenocarcinoma and 39,950 deaths from pancreatic adenocarcinoma occurred in 2014 [2]. The prognosis is dismal with only $10 \%$ to $30 \%$ of pancreatic adenocarcinomas resectable at the time of presentation, a 5 -year survival of $10 \%$ to $20 \%$, and median survival of only 10 to 20 months [3]. Pancreatic adenocarcinoma commonly metastasizes to the liver and patients with metastatic disease have a median survival of only 3 to 6 months. Thus, early diagnosis, accurate staging and curative resection offers the best chance of survival [4].

$\mathrm{CT}$ is the most common imaging modality of choice in staging and follow-up of unresectable pancreatic cancer. Tumor treatment response at CT is commonly assessed by tumor size [5-7] or attenuation changes [8-10]. Positron Emission Tomography (PET), however, has been shown to be more accurate for detection of pancreatic cancer in many studies, with overall accuracy of $91 \%$ versus $78 \%$ for contrast-enhanced CT [11] with comparable sensitivity [12]. PET is generally more accurate and sensitive for distant metastases and also has prognostic value [13], with higher values correlating with more aggressive disease. Thus, a co-registered PET-CT combines optimally both the metabolic information from PET and the good spatial resolution of CT.

Pancreatic cancer is a lethal disease and more precise pretreatment assessment could determine the optimal therapy or identify early

*Corresponding author: Teresa Wu, School of Computing, Informatics, Decision Systems Engineering, Arizona State University, Tempe, 85287, Arizona, USA, Tel: 1-480-965-4157; E-mail: teresa.wu@asu.edu

Received November 03, 2014; Accepted November 20, 2014; Published November 25, 2014

Citation: Zhang M, Oldan J, He M, Wu T, Silva A, et al. (2015) Pilot Study: Two-Stage Hybrid Model to Correlate Single Energy CT and PET in Pancreatic Adenocarcinoma. J Health Med Informat 6: 175. doi:10.4172/2157-7420.1000175

Copyright: $\odot 2015$ Zhang M, et al. This is an open-access article distributed under the terms of the Creative Commons Attribution License, which permits unrestricted use, distribution, and reproduction in any medium, provided the original author and source are credited. 
response or non-response. If this information was reliably obtained, it could allow rapid treatment changes to improve patient outcomes. In a 2006 study of 102 patients, PET had higher diagnostic accuracy than CT (95\% vs 76\%) for pancreatic adenocarcinoma and was also superior in detecting treatment response (5 vs $0 / 15$ cases) [14]. Both Friess et al. [15] and Van Heertum et al. [16] report that for lesions less than $2-\mathrm{cm}$ in diameter, the sensitivity of PET is far superior to that of CT, especially for hypermetabolic tumors. Yet in the cases where the tumor is greater than $4 \mathrm{~cm}$ in diameter, CT outperforms PET. Although some studies indicate that PET may provide superior information than CT for pancreatic adenocarcinoma, CT is most commonly used to detect and follow patients with pancreatic cancer. PET-CT is less commonly used due the higher cost and lower access compared to CT alone.

Given the higher cost and lower access of PET, it would be ideal if one could derive accurate metabolic information with CT alone as is derived at PET. To date, the most commonly used CT biomarkers for pancreatic cancer are the tumor density (i.e., attenuation), tumor size and signal-to-noise ratio (SNR). Prior attempts to correlate specific imaging biomarkers (in both MR and CT) with PET have been done in non-pancreatic tumors such as non-small-cell lung cancer $(r=0.786)$ [17], rectal cancer $(r=0.587)$ [18], nasopharyngeal cancer $(r=-0.372)$ [19], and head and neck tumors $(r=-0.538)$ [20]. A stronger correlation with PET in the case of pancreatic cancer would be useful in assessment of pancreatic tumor pathology and response, and might even allow for substitution of CT for PET-CT in some cases. The goal of this study was to identify the CT biomarker(s) of pancreatic cancers which correlates best with PET.

\section{Materials and Methods}

This retrospective study was performed in accordance with institutional review board guidelines and approval. Informed consent was waived due to the minimal risk of this retrospective review.

\section{Patient selection}

A registry of pancreatic adenocarcinoma patients was reviewed to identify those with PET-CT scans performed within one week of a contrast-enhanced abdominal CT. A total of 24 patients were identified that fit study criteria, 17 males and 7 females. Average age was 65 years, with standard deviation of 9.8 and range of 47 to 86 . Average tumor size was $4.2 \mathrm{~cm}$, with standard deviation of 1.9 and range of 1.5 to 8.4 $\mathrm{cm}$. Average PET SUV ${ }_{\text {max }}$ was 5.3, with standard deviation of 2.4 and range of 1.7 to 12 . Pancreatic cancers were located in the following regions: uncinate process $(n=4)$, head $(n=7)$, neck/body $(n=10)$ and tail $(\mathrm{n}=8)$ (some cancers were present in more than one region). All patients had unresectable or metastatic disease at the time of the CT. Of the 24 patients, 13 had hepatic metastases, 12 nodal metastases, and 21 vascular involvement of the mesenteric vessels or portal vein. All were pathologically proven to be pancreatic adenocarcinoma. 13 patients had had prior chemotherapy and 11 were baseline studies. Twelve of 24 patients (50\%) had cystic areas of the tumor.

\section{CT protocol}

All CTs were performed on a 64-slice CT scanner (CT750 HD, GE Healthcare, Milwaukee, WI) as part of a standard biphasic pancreatic CT protocol. This protocol consisted of a pancreatic phase (approximately $40 \mathrm{sec}$ after contrast injection) and a portal venous phase (approximately $70 \mathrm{sec}$ after contrast injection) after injection of a body-weight-based volume $(1 \mathrm{cc} / \mathrm{kg})$ of low-molecular-weight nonionic iodinated contrast medium (Omnipaque 350, General Electric, Milwaukee, WI) at $4 \mathrm{cc} /$ second. Specific imaging parameters are shown in (Table 1).

\section{PET protocol}

All PET imaging was performed on a combined 16 slice PET/ CT scanner (Discovery PET Scanner, GE Healthcare, Milwaukee, WI) within one week of the enhanced CT scan. The PET (18F-FDG) scan used $3.27 \mathrm{~mm}$ slices and a 3D acquisition with the VUE Point HD reconstruction filter. Imaging matrix was 192 by 192 pixels. Seven to nine $15-\mathrm{cm}$ bed positions were acquired, at 2 minutes per bed position if BMI (body mass index) was less than 35,3 minutes per bed position was applied otherwise. A simultaneous non-contrast CT was performed at $129 \mathrm{kVp}, 100-120 \mathrm{~mA}$, non-helical, with slice thickness being $3.75 \mathrm{~mm}$. Corregistration between PET and non-contrast CT was performed using MIMvista software (MIMvista 5.2.3, by MIM Software Inc., Cleveland, OH). The PET SUV ${ }_{\max }$ value within the tumor was used for analysis.

\section{CT and PET measurements and derived variables}

The location and maximal axial tumor size was recorded. The presence and location of metastatic disease was noted. Circular regions of interest (ROI) were placed to include as much of the designated region as possible (Figure 1). Six measures were initially obtained from the ROIs (Table 2a): the mean CT attenuation of the normal pancreas, solid tumor, upper abdominal aorta, cystic tumor, and the standard deviation of the CT attenuation of normal pancreas, solid tumor. Note for cystic tumor, if no cystic tumor area existed, the cystic variable was set equal to the non-cystic tumor variable. All measurements were obtained by a board-certified radiologist on a commercially available offline computer workstation (GE Advantage Workstation, GE Healthcare, version 4.4). The original 6 measurements were used to derive 10 more biomarkers representing different combination ratios and SNR measurements (Table $2 \mathrm{~b}$ ). Two of the derived biomarkers used the originally measured aortic mean attenuation to normalize the enhancement values of the pancreas, i.e. to minimize the effect of scan timing on the enhancement values. Other derived biomarkers included two different SNR techniques using either the solid or cystic tumor

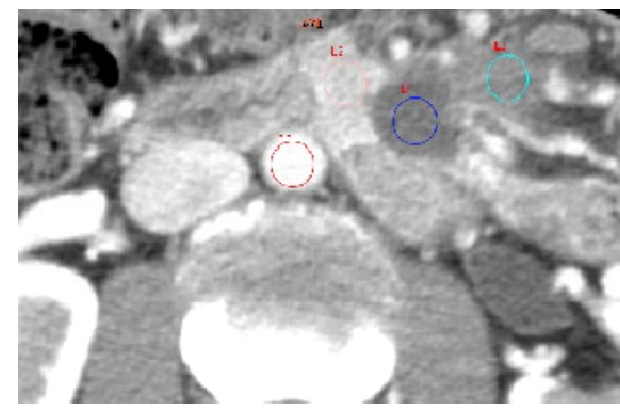

Figure 1: Red, pink, cyan, and blue circles denote ROls of aorta, pancreas, solid tumor, and cystic tumor respectively.

\begin{tabular}{|c|c|c|c|c|c|c|c|}
\hline Series & Speed (mm/rot) & Pitch & Collimation (mm) & Slice Thickness (mm) & Reconstruction Interval (mm) & $k V p$ & $\min / \max \mathrm{mA}$ \\
\hline Pancreatic & \multirow{2}{*}{39.375} & \multirow{2}{*}{0.984} & \multirow{2}{*}{.625} & 2.5 & 2 & 120 & $150-450$ \\
\hline Portal venous & & & & 3.75 & 3 & 120 & 630 \\
\hline
\end{tabular}

Table 1: CT imaging protocol. 
Citation: Zhang M, Oldan J, He M, Wu T, Silva A, et al. (2015) Pilot Study: Two-Stage Hybrid Model to Correlate Single Energy CT and PET in Pancreatic Adenocarcinoma. J Health Med Informat 6: 175. doi:10.4172/2157-7420.1000175

Page 3 of 6

\begin{tabular}{|c|c|c|}
\hline Variables & Measured & Description \\
\hline Solid tumor & Mean HU value of tumor & Direct measurement \\
\hline Cystic tumor & Mean HU value of cystic tumor & Direct measurement \\
\hline Aorta & Mean HU value of aorta & Direct measurement \\
\hline Normal pancreas & Mean HU value of pancreas & Direct measurement \\
\hline Normal pancreas SD & Standard deviation of HU value at pancreas & Direct measurement \\
\hline Solid tumor SD & Standard deviation of HU value at tumor & \\
\hline
\end{tabular}

$\mathrm{SD}=$ standard deviation

Table 2a: CT measurement-6 Original Readings as Imaging Biomarkers.

\begin{tabular}{|l|l|}
\hline Variables & Derived \\
\hline Ratio I & Mean HU value of tumor/mean HU value of pancreas \\
\hline Ratio II & Mean HU value of cystic tumor/mean HU value of normal pancreas \\
\hline Ratio III & Mean HU value of solid tumor /mean HU value of cystic tumor \\
\hline SD Ratio I & Standard deviation of HU value at solid tumor/mean HU value at normal pancreas \\
\hline SD Ratio II & Standard deviation of HU value at solid tumor/standard deviation of pancreas \\
\hline SD Ratio III & Standard deviation of HU value at solid tumor/mean HU value at aorta \\
\hline Normalized enhancement I & Mean HU value of solid tumor/ mean HU value of aorta \\
\hline Normalized enhancement II & Mean HU value of cystic tumor/mean HU value of aorta \\
\hline Signal to Noise Ratio I & (Mean HU value of normal pancreas-mean HU value at solid tumor mean)/Standard Deviation at normal pancreas \\
\hline Signal to Noise Ratio II & (Mean of HU value of normal pancreas-mean HU value at cystic tumor)/Standard Deviation of HU value at normal pancreas \\
\hline
\end{tabular}

Table 2b: CT measurement-10 Derived Readings as Imaging Biomarkers.

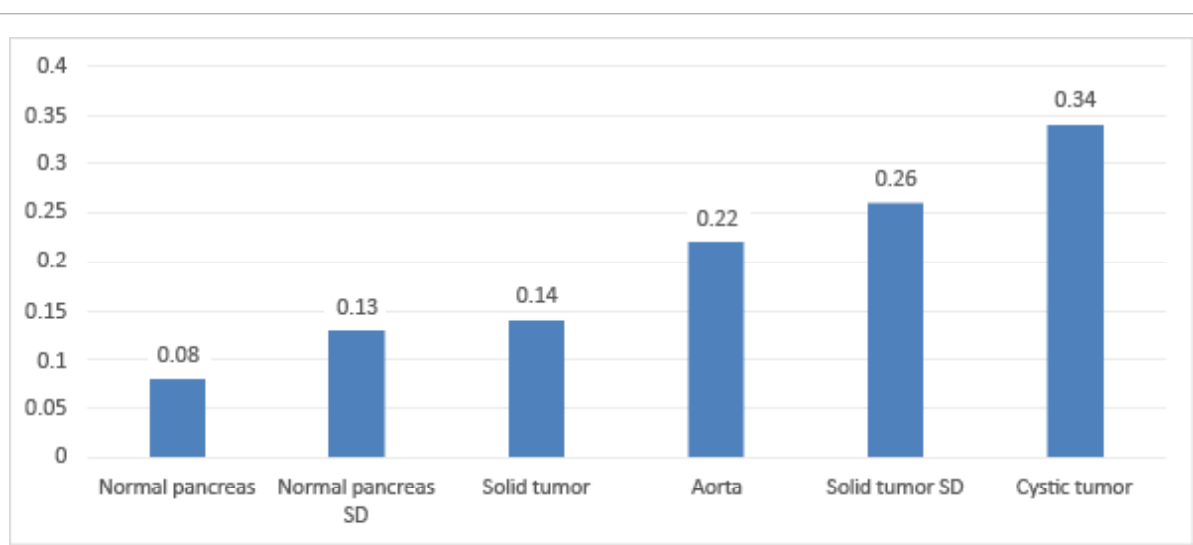

Figure 2a: Univariate analysis on the original 6 measures.

portions.

The response variable, instead of SUV ${ }_{\text {mean }}, S_{\text {max }}$ from PET was measured since in medical practices, $\mathrm{SUV}_{\max }$ has been the standard and SUV ${ }_{\text {men }}$ is not generally used because of its dependence on volume. Indeed. The tumor was identified using registered and fused images on the workstation and the most FDG-avid portion of the tumor identified by visual inspection. Please note in PET, SUV $_{\text {max }}$ was calculated as local concentration $(\mathrm{g} / \mathrm{mCi})$ divided by injected dose $(\mathrm{mCi})$ over weight $(\mathrm{g})$.

\section{Correlation analysis}

Two experiments were conducted: (1) the first experiment was to explore the correlation between PET SUV $\mathrm{max}_{\text {and }}$ and the 6 original biomarkers and the 10 derived biomarkers on all patients. Both univariate analysis (one parameter at a time) and multivariate analysis were conducted. Since the derived biomarkers already captured some nonlinearities between the original biomarkers, first order regression model was studied only; (2) the second experiment was to study the applicability of a two-stage hybrid model to identify the correlations. A multivariate classifier was first developed to group the patient into two cohorts. To identify the appropriate cut-off point from SUVmax to group the patients, we extensively evaluate the performance of the model with SUVmax varied from 2 to 8 . The results indicated SUVmax 5 has the best predictive power. The following experiment was then conducted with two patients cohorts formed based on 5 SUVmax as the cut-off point, which resulted 12 in low and high cohorts, respectively. Next, a multivariate regression analysis was then conducted for each cohort. For both experiments, the statistical modeling was developed using Minitab 16 [Minitab, Inc. (State College, PA)]. The classifier in the second experiment was developed using WEKA [21]. In the multivariate regression analysis, to address the issue of potential multicollinearity among the selected features, the model was developed adding features one at a time. The variance inflation factor (VIF) was then calculated to assess the possible correlations between the features. According to literature [22], VIF $>10$ indicates the model has multicollinearity issue.

\section{Results}

\section{Experiment I: Single stage statistical modeling}

The objective of this experiment was to evaluate the predicative power of simple statistical models using original biomarkers or derived 
biomarkers. For the original 6 biomarkers, univariate analysis was conducted. As shown in Figure 2a, the correlation ( $r$ value) ranges from 0.08 to 0.34 . For the 10 derived biomarkers, the correlation $(r$ value) has wider ranges from 0 to 0.54 . The multivariate analysis identified stronger correlations for both two types of biomarkers. Specifically, the first order regression on the original 6 biomarker has an $r 0.64$ and on the derived biomarker has an $r 0.74$. (The regression model reports $r^{2}$, and so we report $r$-its square root here for a fair comparison.) The details of the regression models are summarized in Table 3. As aforementioned, $\mathrm{p}<0.05$ is used as the threshold to identify the significant factors. In addition, to avoid multicollinearity, VIF $<10$ [22] is used as the guideline to ensure the model has no factors raising multicollinearity issues. Therefore as a result, only 4 out of 6 factors from original CT biomarkers and 2 out of 10 derived factors are selected to build the regression models respectively.

From this experiment, our key findings are three. First, for the original 6 biomarkers, the univariate analysis indicates that the biomarker with the least predictor with $\mathrm{SUV}_{\max }$ is normal pancreas $(\mathrm{r}=0.08)$, the strongest predictor is cystic tumor $(\mathrm{r}=0.34)$. The multivariate regression model (first order) identifies $r$ as 0.64 . The key predictors are cystic tumor (with negative correlation), solid tumor $\mathrm{SD}$, normal pancreas and aorta (with negative correlation). Second, for the 10 derived biomarkers, the univariate analysis indicates signal to noise ratio I has no correlation with $\mathrm{SUV}_{\max }$. Among the remaining 9 biomarkers, the one with the least correlation with SUV $_{\text {max }}$ is Ratio I with an $r=0.17$, the one with the strongest correlation is Ratio III with an $r=0.54$. The first order multivariate regression model identifies $r$ as 0.74 . The key predictors are SD Ratio III and Signal to Noise Ratio II, both with positive correlation. Thirdly, all models present no multicollinearity issue as VIF is $<10$ for all predictors. All predictors are significant with $\mathrm{p}<0.05$.

In summary, the first experiment identified some correlations. As expected, derived biomarkers took advantage of domain knowledge (from CT) did show better correlation outcomes. This motivated us to further explore the domain knowledge from PET, for example, SUV is considered to be high when it is higher than 5 and vice versa, to help the correlation identification. This was done in the second experiment.

\section{Experiment II: Two-stage model on all biomarkers}

All measures including 6 original and 10 derived were considered in this experiment. Initially, there are 6 original factors: Solid tumor, Cystic tumor, Aorta, Normal pancreas, Normal pancreas SD, Solid tumor SD. Since aorta is commonly used to remove the effect of time delay, it was chosen as a denominator. To compare with the normal pancreas, normal pancreas was also chosen as a denominator. This resulted seven factors being investigated in correlation with the $\mathrm{SUV}_{\max }$ : tumor, cyst, tumor/panc, cyst/panc, tumor/cyst, tumor/aorta, cyst/aorta (the panc, aorta are used in the ratio, thus there is no need of them). Similarly, additional six factors are also necessary and are correlated to $\mathrm{SUV}_{\max }$ : SNR (P-T/SDP), SNR (P-C/SDP), SDTumor/ panc, SDtumor/SDPanc, SDtumor/aorta, tumor SD (only tumor SD is needed since panc $\mathrm{SD}$ is used in the derived variables as denominator). As a result, there were total 13 independent biomarkers ( 3 original and 10 derived). First, the patients were grouped into two cohorts using SUV value 5 as the cut-off point. By using those 13 features as inputs, Naïve Bayesian classifier [21] was trained to predict the patients belonging to the group with high SUV value $\left(\mathrm{SUV}_{\text {max }}>5\right.$ ) or low SUV value $\left(\mathrm{SUV}_{\max }<=5\right)$. To avoid overfitting, 10 fold cross validation was implemented. In each of the 10 cross validation run, $10 \%$ of the patients (randomly) were put aside to test the model performance, and the remaining $90 \%$ of the patients were used to develop the classifier. The average performance of the 10 runs was collected and reported. In this study, the classification accuracy was $91 \%$ ( 2 out of 24 patients were wrongly classified). Next, for each group, first order regression model was developed with an $r^{2}=0.86(r=0.93)$ for the high SUV cohort and an $r^{2}=0.68$ ( $\left.r=0.82\right)$ for the low SUV cohort. Considering the two stage together, for all the 24 patients, the overall prediction performance is $r^{2}=0.81(r=0.90)$.

\section{Discussions}

The goal of our study was to determine correlation of single energy

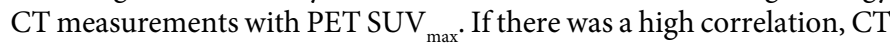
could then potentially be used as an imaging surrogate or complement to PET, saving costs, radiation and inconvenience to the patient. The results of our study found that multivariate analysis using several CT imaging biomarkers correlated better with PET SUV max $_{\text {compared to }}$ univariate correlations such as tumor attenuation or SNR. We also found the derived imaging biomarkers showed stronger correlation compared with the original biomarker.

Overall, tumors with a higher $\mathrm{SUV}_{\max }$ tend to have a more 'aggressive' CT appearance, but this itself may differ from tumor to tumor, and different characteristics of an 'aggressive' appearance (necrosis, angiogenesis, heterogeneity, hyperenhancement) may be salient in each case. It is quite possible that each tumor type may have its own characteristics that correlate with tumor aggressiveness. Generally, a higher SUV ${ }_{\text {max }}$ is a poor prognostic indicator as well, as it

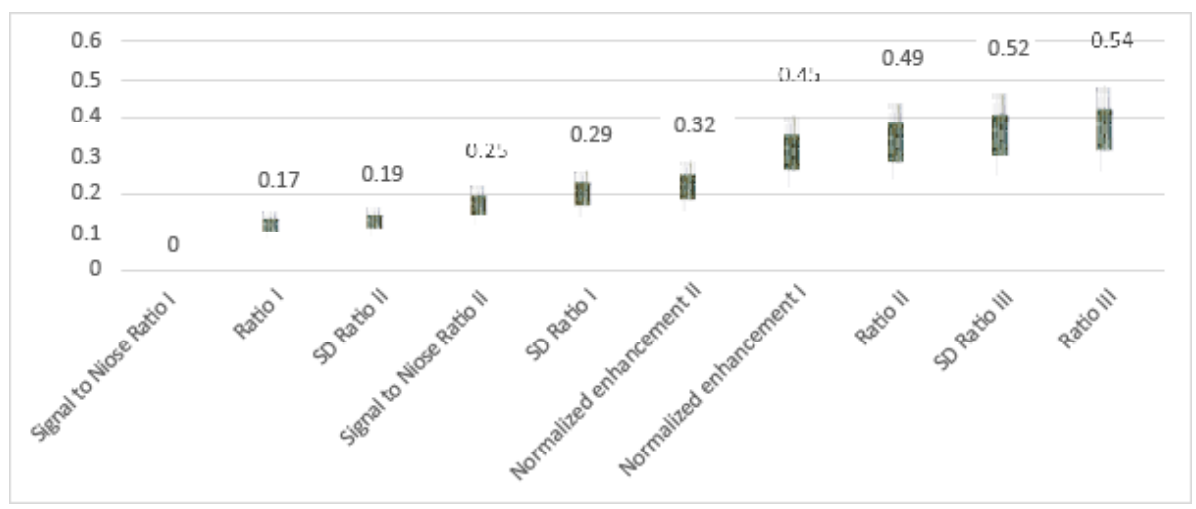

Figure 2b: Univariate analysis on the derived 10 biomarkers. 


\begin{tabular}{|l|c|c|c|}
\hline Predictors & Coefficient & p-value* & VIF $^{* *}$ \\
\hline Cystic tumor & -0.05129 & 0.036 & 1.117 \\
\hline Solid tumor SD & 0.21773 & 0.041 & 1.073 \\
\hline Normal pancreas & 0.04310 & 0.035 & 1.888 \\
\hline Aorta & -0.023097 & 0.032 & 1.909 \\
\hline
\end{tabular}

${ }^{*} p<0.05$ indicate the predictor is statistically significant

${ }^{*} \mathrm{VIF}<10$ indicates the predictor has no multicollinearity issue

Table 3a: First Order Regression model on the original CT imaging biomarkers for SUV $_{\text {max }},\left(r^{2}=0.41, r=0.64\right)$

\begin{tabular}{|l|c|c|c|}
\hline Predictors & Coefficient & p-value* & VIF $^{* *}$ \\
\hline SD Ratio III & 108.33 & 0.000 & 1.220 \\
\hline Signal to Noise Ratio II & 0.7529 & 0.002 & 1.220 \\
\hline
\end{tabular}

${ }^{*} p<0.05$ indicate the predictor is statistically significant

${ }^{*} \mathrm{VIF}<10$ indicates the predictor has no multicollinearity issue

Table 3b: First Order Regression model on the derived CT imaging biomarkers for SUV $_{\text {max }}\left(r^{2}=0.55, r=0.74\right)$

\begin{tabular}{|l|c|c|c|}
\hline Predictors & Coefficient & p-value* & VIF \\
\hline Normalized Enhancement I & 36.1808 & 0.001 & 2.14944 \\
\hline Solid tumor & -0.0706 & 0.004 & 1.58404 \\
\hline Signal to Noise I & 1.0963 & 0.009 & 6.83672 \\
\hline Ratio I & 8.7538 & 0.023 & 6.83934 \\
\hline
\end{tabular}

${ }^{*} p<0.05$ indicate the predictor is statistically significant

${ }^{*} \mathrm{VIF}<10$ indicates the predictor has no multicollinearity issue

Table 4a: First Order Regression model on all CT imaging biomarkers for high SUV $_{\max }\left(\right.$ SUV $\left._{\max }>5\right)$ cohort $\left(r^{2}=0.86, r=0.93\right)$

\begin{tabular}{|l|c|c|c|}
\hline Predictors & Coefficient & p-value* & VIF \\
\hline Cystic tumor & -0.06176 & 0.001 & 1.000 \\
\hline
\end{tabular}

${ }^{*} p<0.05$ indicate the predictor is statistically significant

${ }^{* *} \mathrm{VIF}<10$ indicates the predictor has no multicollinearity issue

Table 4b: First Order Regression model on all CT imaging biomarkers for low SUV $_{\max }\left(\right.$ SUV $\left._{\max }<=5\right)$ cohort $\left(r^{2}=0.68, r=0.83\right)$.

is associated with higher probability of recurrence [23], unresectable disease [24] and lower survival [25-27]. As described previously, in GI stromal tumors [28], an interval decrease in tumor attenuation has correlates with improved prognosis using new targeted therapies, and decreases in enhancement are also important in evaluating hepatocellcular cancer response [29]. Thus, it is possible that some of these new parameters may be of prognostic importance in monitoring pancreatic adenocarcinoma therapy as well.

Since pancreatic tumors with higher SUV may have a different CT appearance compared to those with lower SUV, a machine learning classifier was developed to separate the patients into two cohorts and different regression models were developed for each cohort. Much stronger correlations (overall $r^{2}=0.81, r=0.90$ ) were identified with this two-stage process. We note that to date, most CT studies have relied on simplistic univariate measurements (i.e., tumor density or SNR) to derive prognostic or diagnostic information. The results of our study suggest that more complex CT analytics on these simple measures may still be able to identify good correlation with PET. This has important clinical implications as it implies that valuable information about pancreatic tumors is present at CT but not being optimally analyzed to derive the greatest information.

In the two-stage model, the multivariate classifier (on the 13 biomarkers) was able to group 22 out of 24 into the correct groups (high SUV vs. lower SUV). For the high SUV cohort, the regression model was $r^{2}=0.86$ ( $\left.r=0.93\right)$. The key variables with highest PET SUV ${ }_{\max }$ all involved measurements of the solid portion of the tumor. These included normalized enhancement (the ratio of solid tumor to aorta), solid tumor, ratio of solid tumor to pancreas and signal to noise (mean pancreas-mean solid tumor/standard deviation of pancreas). It appears in the high SUV cases, normalized enhancement which minimizes the effect of scan timing is crucial. In addition, solid tumor density is a key factor, negatively correlating to the $\mathrm{SUV}_{\max }$

For the low SUV cohort, the regression model was $r^{2}=0.68(r=0.83)$. There was only one key variable with high PET SUV ${ }_{\max }$ correlation: the mean attenuation of the cystic tumor (negative correlation). This negative correlation implies that more metabolically active tumors may result in more necrosis. This has also been implied in prior studies where the ratio of metabolism (as measured by FDG-PET) to blood flow (as measured by oxygen-18-water PET) was higher in malignant tumors [30]. Therefore, decreased blood flow leading to necrosis is a feature of metabolically active pancreatic cancers. It is interesting that the attenuation of the solid portion of the tumor was not identified as a key variable in this low SUV group although this is commonly used to assess response in clinical practice. In addition, various ratios or calculated values such as SNR did not improve PET SUV ${ }_{\max }$ correlation. These results suggest that for patients with lower SUV measures, cystic rather than solid tumor density is the key factor to correlate to the PET SUV.

While promising, we do note that one of the principal limitations of our study is a relatively small sample size. A larger sample size would allow us to detect more subtle correlations and assert with greater confidence the correlations we have found. Another limitation is the retrospective nature of the study; a fair amount of heterogeneity exists and it would be interesting to see if patients with prior chemotherapy had different relationships between PET and CT parameters than the patients measured at baseline. Additionally, cystic tumor components were present in only $50 \%$ of cases. Finally, the multivariate analysis was done manually, using non-commercial methods [31].

Our study indicates that with more advanced CT analytics, a two-stage model integrating multivariate machine learning with multivariate regression results in better correlation with $\mathrm{PET} \mathrm{SUV}{ }_{\max }$ than a univariate approach. These techniques have the potential to provide prognostic or treatment information for patients undergoing single energy CT for pancreatic adenocarcinoma that correlate better with PET results. Further studies are needed to confirm these results.

\section{References}

1. American Cancer Society (2014) Cancer facts and figures year 2014 surveillance research from the American Cancer Society. Bethseda (MA).

2. http://www.pancreatic.org/site/c.htJYJ8MPIwE/b.5050503/k.40C9/Pancreatic Cancer Facts.htm,

3. Faria SC, Tamm EP, Loyer EM, Szklark J, Choi H, et al. (2004) Diagnosis and staging of pancreatic tumors. Semin Roentgenol 39: 397-411.

4. Kedra B, Popiela T, Sierzega M, et al. (2001) Prog-nostic factors of long-term survival after respective procedures for pancreatic cancer. Hepatogastroenterology; 48:1762-1766

5. Eisenhauer EA, Therasse P, Bogaerts J,Schwartz LH, Sargent D, et al. (2009) New response evaluation criteria in solid tumours: revised RECIST guideline (version 1.1) Eur J Cancer 45 : 228-247.

6. Rezai P, Yaghmai V, Tochetto SM, Galizia MS, Miller FH, et al. (2011) Change in the growth rate of localized pancreatic adenocarcinoma in response to gemcitabine, bevacizumab, and radiation therapy on MDCT. Int J Radiat Oncol Biol Phys 81: 452-459.

7. Patel M, Hoffe S, Malafa M, Hodul P, Klapman J, et al. (2011) Neoadjuvant 
Citation: Zhang M, Oldan J, He M, Wu T, Silva A, et al. (2015) Pilot Study: Two-Stage Hybrid Model to Correlate Single Energy CT and PET in Pancreatic Adenocarcinoma. J Health Med Informat 6: 175. doi:10.4172/2157-7420.1000175

Page 6 of 6

GTX chemotherapy and IMRT-based chemoradiation for borderline resectable pancreatic cancer. J Surg Oncol. 104: 155-161.

8. Galvin A, Sutherland T, Little AF (2011) Part 1: CT characterisation of pancreatic neoplasms: a pictorial essay. Insights Imaging 2: 379-388.

9. Miura F, Takada T, Amano H, Masahiro Y, Shigeru F, et al. (2006) Diagnosis of pancreatic cancer. HPB (Oxford) 8: 337-342

10. Lee H, Lee JK, Kang SS, Choi D, Jang KT, et al. (2007) Is there any clinical or radiologic feature as a preoperative marker that can differentiate chronic pancreatitis from early-stage pancreatic adenocarcinoma ? Hepatogastroenterology. 54: 2134-2140.

11. Gaetano DAM, Rufini V, Castaldi P, Gatto AM, Filograna L, et al. (2012) Clinical applications of (18)F-FDG PET in the management of hepatobiliary and pancreatic tumors. Abdom Imaging 37: 983-1003.

12. Lan BY, Kwee SA, Wong LL (2012) Positron emission tomography in hepatobiliary and pancreatic malignancies: a review. Am J Surg 204: 232-241.

13. Epelbaum R, Frenkel A, Haddad R, Sikorski N, Strauss LG, et al. (2013) Tumo aggressiveness and patient outcome in cancer of the pancreas assessed by dynamic 18F-FDG PET/CT. J Nucl Med. ;54:12-18.

14. Bang S, Chuang HW, Park SW, Chuang JB, Yun M (2006) The clinical usefulness of 18-fluorodeoxyglucose positron emission tomography in the different diagnosis, staging, and response evaluation after concurrent chemoradiotherapy for pancreatic cancer. J Clin Gastroenterol 40: 923-929.

15. Friess H, Langhans J, Ebert M, Beger HG, Stollfuss J, et al. (1995) Diagnosis of pancreatic cancer by 2(18F)-fluoro-2-deoxy-D-glucosepositron emission tomography. Gut 36:771-777.

16. Van Heertum RL, Fawwaz RA (2001)The role of nuclear medicine in the evaluation of pancreatic disease. Surg Clin North Am 81: 345-358.

17. Schmid BG, Henzler T, Chu TQ, Meyer M, Nance JW et al. (2012) Functional imaging of lung cancer using dual energy CT: how does iodine related attenuation correlate with standardized uptake value of 18FDG-PET-CT? Eur Radiol 22: 93-103.

18. Schmid BG, Henzler T, Chu TQ, Meyer M, Nance JW et al. (2012) Functiona imaging of lung cancer using dual energy CT: how does iodine related attenuation correlate with standardized uptake value of 18FDG-PET-CT? Eur Radiol 22: 93-103.

19. Huang B, Chan T, Kwong DL, Chan WK, Khong PL et al. (2012) Nasopharyngea carcinoma: investigation of intratumoral heterogeneity with FDG PET/CT. AJR Am J Roentgenol 199:169-174.
20. Hirasawa S, Tsushima Y, Takei H, Hirasawa H, Taketomi TA et al. (2007) Inverse correlation between tumor perfusion and glucose uptake in human head and neck tumors. Acad Radiol 14: 312-318.

21. Hall M, Eibe F, Holmes G, Pfahringer B, Reutemann P (2009) The WEKA data mining software: An update. SIGKDD Explorations 11:10-18.

22. Montgomery DC, Peck EA, Geoffrey G (2006) Introduction to Linear Regression Analysis, 4th (Fourth) Edition. New York, NY: Wiley-Interscience.

23. Okamoto K, Koyama I, Miyazawa M, Toshimitsu Y, Aikawa M, et al. (2011) Preoperative 18[F]-fluorodeoxyglucose positron emission tomographycomputed tomography predicts early recurrence after pancreatic cancer resection. Int J Clin Oncol 16: 39-44.

24. Wakabayashi H, Nishiyama Y, Otani T, Toshimitsu Y, Aikawa M, et al. (2008) Role of $18 \mathrm{~F}$-fluorodeoxyglucose positron emission tomography imaging in surgery for pancreatic cancer. World J Gastroenterol. 14: 64-69.

25. Maemura K, Takao S, Shinchi H, Noma H, Mataki Y, et al. (2006) Role of positron emission tomography in decisions on treatment strategies for pancreatic cancer. J Hepatobiliary Pancreat Surg. 13: 435-441.

26. Sperti C, Pasquali C, Cherichetti F, Ferronato A, Decet G, et al. (2003)18-Fluorodeoxyglucose positron emission tomography in predicting survival of patients with pancreatic carcinoma. J Gastrointest Surg 7: 953-959.

27. Nakata B, Nishimura S, Ishikawa T, Ohira M, Nishino H, et al. (2001) Prognostic predictive value of $18 \mathrm{~F}$-fluorodeoxyglucose positron emission tomography for patients with pancreatic cancer. Int J Oncol 19: 53-58.

28. Choi H, Charnsangavej C, Faria SC, Macapinlac HA, Burgess MA, et al. (2007) Correlation of computed tomography and positron emission tomography in patients with metastatic gastrointestinal stromal tumor treated at a single institution with imatinib mesylate: proposal of new computed tomography response criteria. J Clin Oncol. 25: 1753-1759.

29. Lencioni R, Llovet JM (2010) Modified RECIST (mRECIST) assessment for hepatocellular carcinoma. Semin Liver Dis 30: 52-60.

30. Hundt W, la Fougère C, Vogtmann J, Steinbach S, Burbelko M, et al. (2012) Evaluation of contrast medium enhancement and [(18)F]-FDG uptake of liver metastasis in PET/CT prior to therapy. Eur J Radiol 81: 652-657.

31. Shastry M, Miles KA, Win T, Janes SM, Endozo R, et al. (2012) Integrated $18 \mathrm{~F}$-fluorodeoxyglucose-positron emission tomography/dynamic contrastenhanced computed tomography to phenotype non-small cell lung carcinoma Mol Imaging 11: 353-360. 